\title{
Design of the Ammunition Degreasing Based on PLC in the Ammunition Preparation Operation System
}

\author{
Lijuan Cai \& Wei Ge \\ Changchun University of Science \& Technology, Changchun 130022, China \\ E-mail: juanlicai@sina.com.cn
}

Received: July 5, 2011

Accepted: August 22, 2011

doi:10.5539/mas.v5n5p244

\begin{abstract}
Before the ammunitions are used, repaired, and discarded, the oil layers on the surface must be eliminated, and the ammunition oiling can obviously enhance the antirust and sealing property, and reduce the influences of the temperature and the humidity change, but the ammunition oiling must be eliminated before the ammunitions are used. At present, the degreasing methods mainly include the physical heating, mechanical abrasion, chemical cleaning, and high pressure water washing, and to adopt PLC to control the degreasing of the ammunition preparation operation system could enhance the working efficiency and the safety coefficient of the ammunition test, reduce test personal's labor intensity, and realize the automatization or semi-automatization of the ammunition preparation operation.
\end{abstract}

Keywords: Ammunition preparation, Automatic transmission, Degreasing, PLC

The ammunition degreasing is the key of the preparation operation, and the former traditional degreasing method is to use the flaxes repeatedly scrub and wipe out the ammunition by dipping in kerosene or turpentine. This method wastes human resources and materials, with low working efficiency. Through repeating demonstrations and sufficient survey, the greasing method of using PLC to control the high pressure water is selected in this article.

\section{Components}

The equipment is mainly composed by the rotating clamping system, the pneumatic sealing system, the high pressure water washing system, the flushing-prevent system, the compressed air system, and the case (seen in Figure 1).

\subsection{Rotating clamping system}

This system is composed by the principal axis drum, the internal spline rotating axis, the external spline clamp, fixed cylinder, and the motor.

Two rotary sleeves have cone chucks, and their poles have external spline clamps, fixed cylinder, and motor.

\subsection{Pneumatic sealing system}

This system is designed to close the greasing box, and it is composed by two cylinders, two flexible sealing doors, and rotating pins.

\subsection{High pressure water washing system}

This system is mainly used to eliminate the oils on the pill surface, and it is composed by the water box, the high pressure pump, the pressure valve, the selector valve, and the high pressure water nozzle. The working pressure can be adjusted by the pressure valve, and the close and open can be controlled by the selector valve. When the pressure achieves about $10 \mathrm{Mpa}$, the high pressure waters rush to the oiling surface of pills, and eliminate the oiling in short time, and the sewages could be repeatedly used through filtration, if the quantity is large, and if the quantity less, the sewages could be discharge into the ground.

\subsection{Oil-water flushing-prevent system}

When the nozzle of the high pressure water begins spraying water and degreasing oils, if the water on the ammunition and the oils flushed by the water have not been controlled or limited, these waters and oils will splashed to other places of the ammunition, causing secondary pollution, so these oil waters must be drained. The gas-current-limiting is used to control the waste water. On two ends of the ammunition body, there or two or four gas jets, and when the high pressure water begins degreasing, the flushing-prevent system begins to work, 
and these gas jets begin to spray high pressure air current to the outside, forming two gas screens on two ends of the ammunition body, and these two gas screens can effectively prevent the water current and the eliminated oils flow to the place without oils, so the flushing of sewages can be prevented sufficiently.

\subsection{Compressed air system}

When the degreasing station begins to work, a pressure-consistent gas source needs to be equipped to supply the compressed airs. Furthermore, the clamping equipment of the washing machine, the oil water flushing-prevent system, and the sealing door system all need the compressed air as the power.

\section{Working principle}

Except for the water system, other devices of the degreasing system are all placed in the sealing box with transparent observation window to prevent the pollution to the work environment when the high pressure water works.

The procedures of the degreasing work can be described as follows.

(1) By the push of the hydro-cylinder, the ammunition delivering dollyl delivers the ammunitions to the degreasing station, and sealing door opens, and the system puts the ammunitions into the degreasing box from the vertical direction.

(2) The rotating clamping system begins to work, and clamps the pills, and the V-shape base of the dolly descends, and the sealing door closes.

(3) The electromagnetic clutch of the motor in the clamping system works and drives the rotating axis to rotate.

(4) The flushing-prevent jet system begins to work, and form two gas screens on two ends of the ammunition body.

(5) The high pressure pump works and begins to wash the pills.

(6) After certain time, the high pressure water stops to spray, and the compressed air begins to blow until the pills are dried incompletely.

(7) The flushing-prevent system stops working.

(8) The ammunition delivering dolly 2 rises from the box, and the sealing board opens, and the system delivers the washed pills to the next station, so the first pill is cleared completely. And when the first pill is delivered to the next station, the dolly 1 delivers the second pill for degreasing.

Above procedures are repeated and the degreasing work can be completed.

\section{Electrical apparatus control system of ammunition degreasing station}

\subsection{Working principle of control system}

When the first pill is put on the dolly 1 , the control system controls the hydraulic system and delivers the dolly 1 to the degreasing station, and the V-shape base rises, and the pneumatic sealing door opens, and the clamping system begins to work, and the V-shape base descends, and the sealing door closes, and the motor starts and drives the roller to rotate. At the same time, the magnetic valve starts, and the high pressure jet begins to spray high pressure water to the pills, and wash the oils on the surface of pills. After certain time, the motor stops, and the high pressure water magnetic valve acts, and the high pressure water stops spraying, and the gas jets are started to blow the pills.

\subsection{System chart of the electrical apparatus control system of ammunition degreasing station}

The system chart of the electrical apparatus control system of ammunition degreasing station is seen in Figure 2.

\section{$3.3 \mathrm{I} / O$ point distribution of PLC function system}

According to the design of input and output, 13 input points and 25 output points are needed, and the PLC of $\mathrm{FX}_{2}$-64MR is selected, and its output form is the relay output, which can drive the $\mathrm{AC}$ or DC load, with 32 input points and 32 output points, so it can satisfy the design requirements, and the concrete I/O address distribution is seen in Table 1.

\subsection{Design of the ammunition degreasing station control circuit}

(1) Detect the level of water box.

(2) Detect the air pressure signal of pneumatic pump.

(3) Detect the water pressure signal of high pressure pump. 
(4) Detect the pill signal of V-shape base 1.

(5) Give the signal of opening the oil box door.

(6) Detect whether the oil box door is opened.

(7) Give the rising signal of V-shape 1.

(8) Detect whether the pill is clamped tightly.

(9) Give the clamping signal of the clamping system.

(10) Detect the pill is clamped tightly.

(11) Give the descending signal of the V-shape base 1.

(12) Give the signal of closing the oil box door.

(13) Start the rotating motor.

(14) Give the flushing-prevent jet signal.

(15) Start the high pressure water magnetic valve.

(16) Close the high pressure water magnetic valve after $5 \mathrm{sec}$.

(17) Give the magnetic valve signal of gas nozzle.

(18) Give the signal of closing the flushing-prevent system.

(19) Give the signal of closing the rotating motor.

(20) Give the magnetic valve signal of closing the gas nozzle.

(21) Give the signal of opening the oil box.

(22) Detect whether the dolly 2 achieves the station 1 .

(23) Give the rising signal of V-shape base 2.

(24) Give the signal of closing the clamping system.

(25) Give the ascending signal of V-shape base 2.

(26) Give the signal of closing the oil box.

\subsection{Design of the PLC control flow chart}

According to the design task, PLC should satisfy following functions.

(1) When the pill rises, the sensor under the box detects the signals, and the system opens the sealing door, and put the pill from the vertical direction into the degreasing box.

(2) The rotating clamping system clamps the pill, and the V-shape base of the dolly descends, and the sealing door closes.

(3) The electromagnetic clutch of the motor in the clamping system works and drives the rotating axis to rotate.

(4) The flushing-prevent jet system begins to work, and form two gas screens on two ends of the ammunition body.

(5) The high pressure pump works and begins to wash the pills.

(6) To the time of t, the high pressure water stops to spray, and the compressed air begins to blow until the pills are dried incompletely.

(7) The flushing-prevent system stops working.

(8) The ammunition delivering dolly 2 rises from the box, and the sealing board opens, and the system delivers the washed pills to the next station.

The flow chart of the design is seen in Figure 3.

\section{Conclusions}

The ammunition degreasing is the key of the preparation operation, and the former traditional degreasing method is to use the flaxes repeatedly scrub and wipe out the ammunition by dipping in kerosene or turpentine. This method wastes human resources and materials, with low working efficiency. Through repeating demonstrations and sufficient survey, the greasing method of using PLC to control the high pressure water is selected in this article. This method could enhance the working efficiency and the safety coefficient of the ammunition test, 
reduce test personal's labor intensity, and realize the automatization or semi-automatization of the ammunition preparation operation.

\section{References}

Cai, Lijuan, Cai, Lijing \& Li, Yang. (2009). The Design of Ammunition Preparation Operating System Based on PLC. Equipment Manufacturing Technology. No.7.

Gao, Fuhua \& Yang, Xiaoping. (1999). Electro-technics. Beijing: China Machine Press.

Gong, Zongren, Wu, Yifeng \& Guo, Yong. (2002). PLC Application System Design and Communication Network Technology. Beijing: The People's Posts and Telecommunications Press. Sep of 2002.

Guo, Zongren, Wang, Zhikai \& Li, Yan. (2002). The Application and Realization of PLC Hierarchical Intelligent Control System. Acta Electronica Sinica. No.4.

Liao, Changchu. (2002). PLC Programming and Application. Beijing: China Machine Press. Sep of 2002.

Liu, Peng'an, Huang, Pengbo, You, Wei \& Yao, Rongguo. (2010). Application of High Pressure Water Jet in Ammunition Surface Degreasing. Surface Technology. No.1.

Wang, Renxiang. (2001). Common-used Low-voltage Apparatus Principle and Control Technology. Beijing: China Machine Press.

Wang, Tianmiao \& Chou, Wusheng. (2002). Electromechanical Control Basic Theory and Application. Beijing: Tsinghua University Press. 
Table 1. I/O address distribution of degreasing station

\begin{tabular}{|c|c|c|c|}
\hline IN Point No. & Input Signals & $\begin{array}{l}\text { OUT Point } \\
\text { No. }\end{array}$ & Output Control \\
\hline X001 & System starting signal & Y401 & $\begin{array}{l}\text { Open the inlet valve control } \\
\text { switch }\end{array}$ \\
\hline X002 & $\begin{array}{c}\text { Water box water low level } \\
\text { detection signal }\end{array}$ & Y402 & $\begin{array}{l}\text { Close the inlet valve control } \\
\text { switch }\end{array}$ \\
\hline X003 & $\begin{array}{c}\text { Water box water high level } \\
\text { detection signal }\end{array}$ & Y403 & Open the barometric switch \\
\hline X004 & $\begin{array}{c}\text { Air pressure lower limit detection } \\
\text { signal }\end{array}$ & Y404 & Close the barometric switch \\
\hline X005 & $\begin{array}{c}\text { Air pressure higher limit detection } \\
\text { signal }\end{array}$ & Y405 & $\begin{array}{c}\text { Open the water pressure control } \\
\text { switch }\end{array}$ \\
\hline X006 & $\begin{array}{c}\text { Water pump water pressure lower } \\
\text { limit detection signal }\end{array}$ & Y406 & $\begin{array}{l}\text { Close the water pressure control } \\
\text { switch }\end{array}$ \\
\hline X007 & $\begin{array}{l}\text { Water pump water pressure higher } \\
\text { limit detection signal }\end{array}$ & Y407 & Enabling signal of oil box door \\
\hline X008 & Dolly 1 position detection signal & Y408 & Rising signal of V-shape base 1 \\
\hline X009 & Dolly 1 pill detection signal & Y409 & Stop signal of V-shape base 1 \\
\hline $\mathrm{X} 010$ & $\begin{array}{l}\text { V-shape base } 1 \text { position detection } \\
\text { signal }\end{array}$ & Y410 & $\begin{array}{l}\text { Clamping signal of clamping } \\
\text { cylinder }\end{array}$ \\
\hline X011 & Pill clamping detection signal & Y411 & $\begin{array}{c}\text { Descending signal of V-shape } \\
\text { base } 1\end{array}$ \\
\hline $\mathrm{X} 012$ & Dolly 2 position detection signal & Y412 & Shutdown signal of oil box door \\
\hline \multirow[t]{13}{*}{ X013 } & $\begin{array}{c}\text { V-shape base } 2 \text { position detection } \\
\text { signal }\end{array}$ & Y413 & Starting signal of rotating motor \\
\hline & & Y414 & $\begin{array}{c}\text { Starting system of } \\
\text { flushing-prevent system }\end{array}$ \\
\hline & & Y415 & $\begin{array}{c}\text { Starting signal of high pressure } \\
\text { water electromagnetic valve }\end{array}$ \\
\hline & & Y416 & $\begin{array}{l}\text { Stop signal of high pressure } \\
\text { water electromagnetic valve }\end{array}$ \\
\hline & & Y417 & Timer \\
\hline & & Y418 & $\begin{array}{c}\text { Starting signal of compressed } \\
\text { air source }\end{array}$ \\
\hline & & Y419 & $\begin{array}{l}\text { Stop signal of flushing-prevent } \\
\text { system }\end{array}$ \\
\hline & & Y420 & Stop signal of rotating motor \\
\hline & & Y421 & $\begin{array}{c}\text { Stop signal of compressed air } \\
\text { source }\end{array}$ \\
\hline & & Y422 & Rising signal of V-shape Base 2 \\
\hline & & Y423 & $\begin{array}{l}\text { Cutoff signal of clamping } \\
\text { cylinder }\end{array}$ \\
\hline & & Y424 & $\begin{array}{c}\text { Descending signal of V-shape } \\
\text { base } 1\end{array}$ \\
\hline & & Y425 & System output signal \\
\hline
\end{tabular}




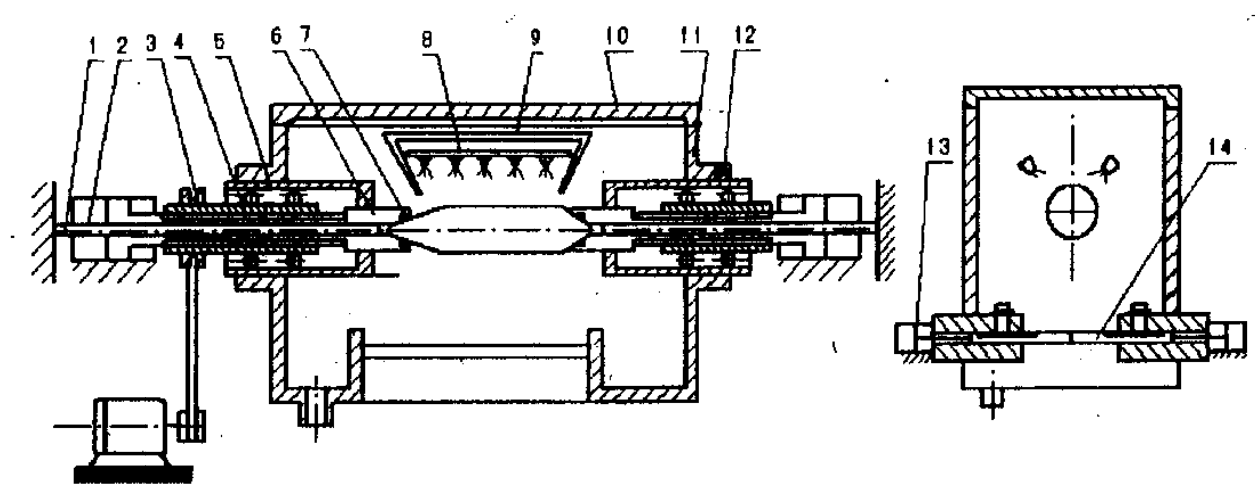

Note: 1-buting iron, 2-fixed cylinder, 3-belt pulley, 4-internal spline rotating shaft, 5-principal axis drum, 6-external spline clamp, 7-seal ring, 8-high pressure water jet, 9-flushing-prevent gas screening case, 10-case cover, 11-case body, 12-gear rack, 13-cylinder, 14-pneumatic seal pad

Figure 1. Structure Diagram of Ammunition Degreasing Device

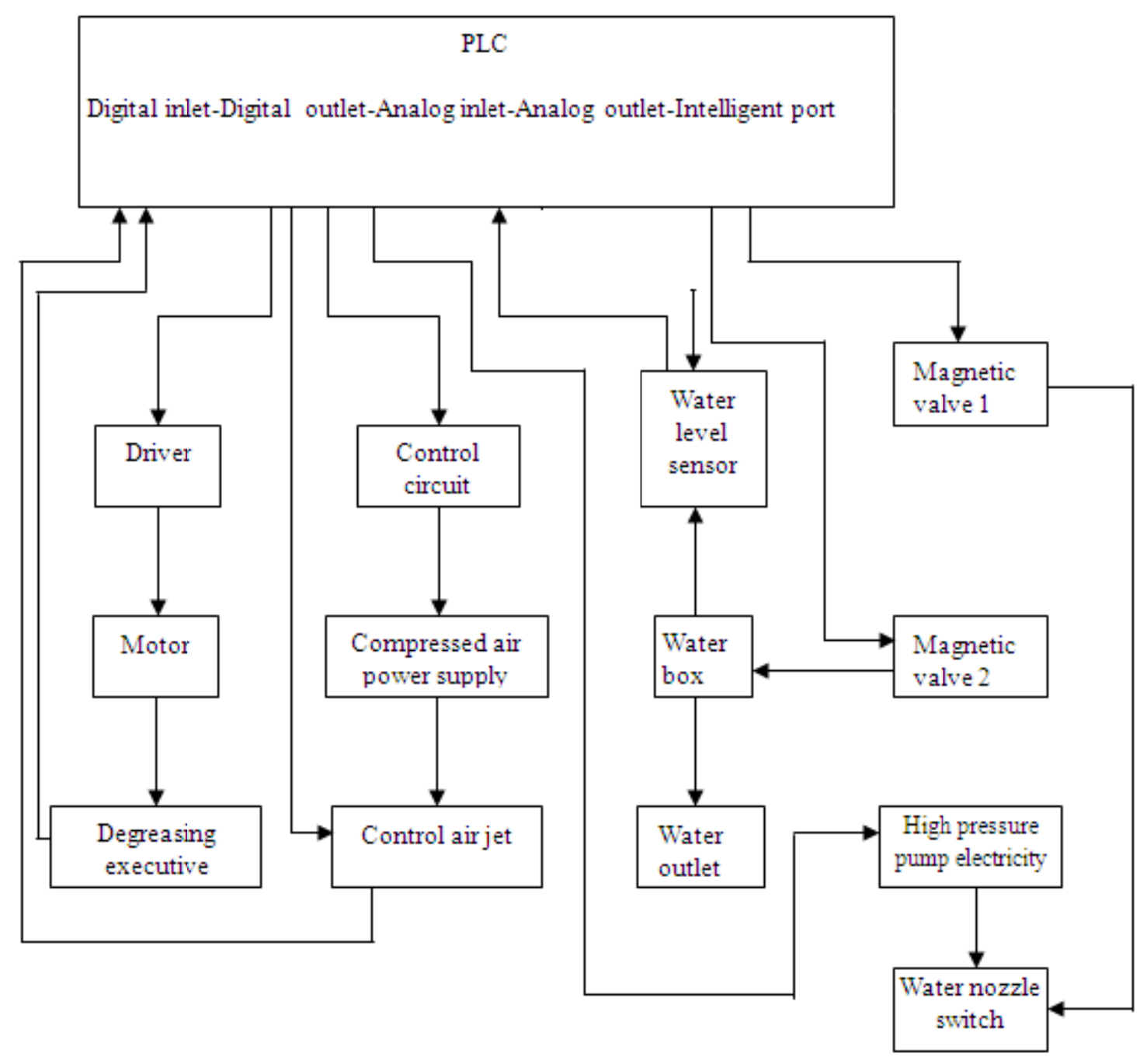

Figure 2. Control System Chart of Ammunition Degreasing Station 


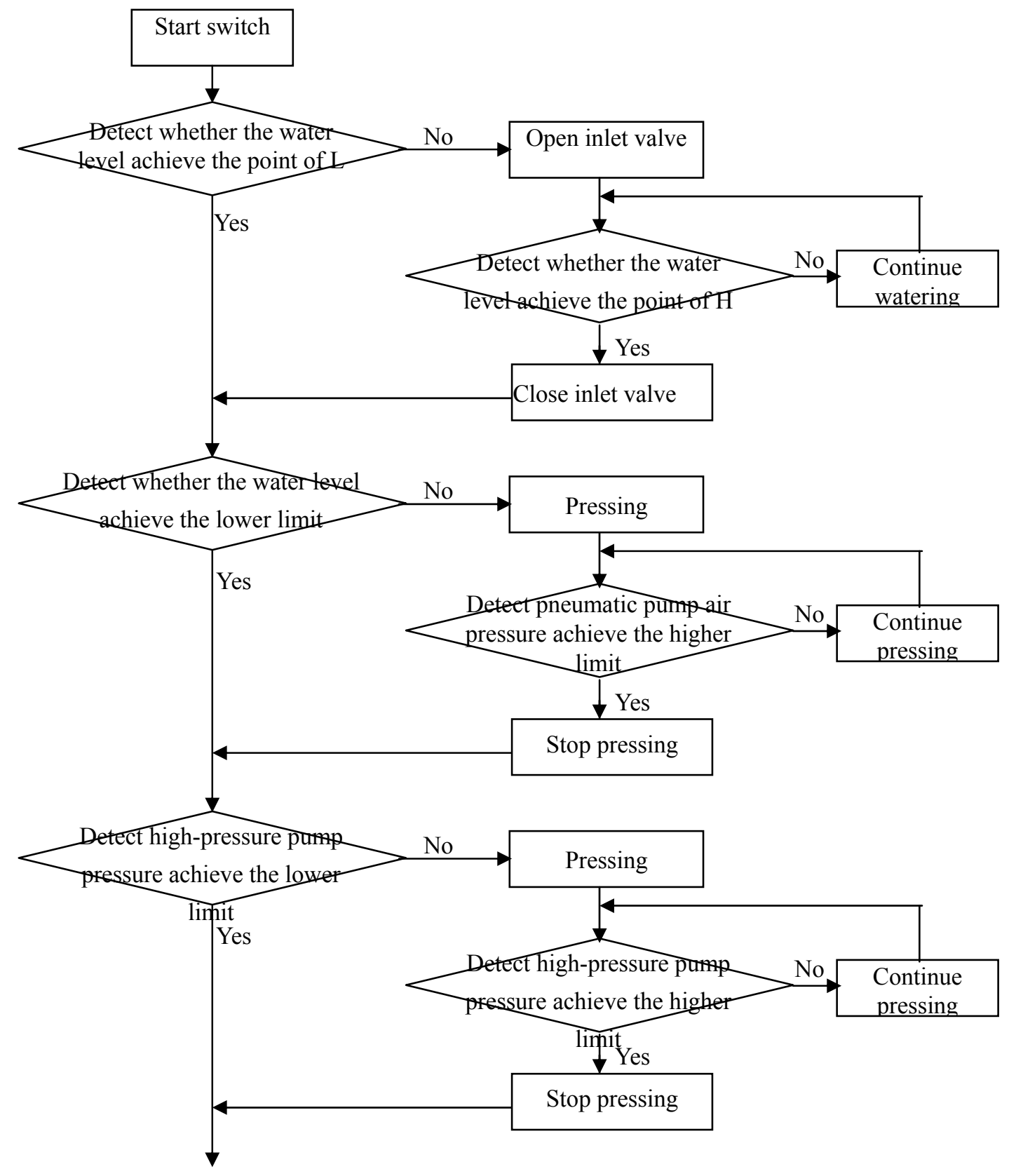




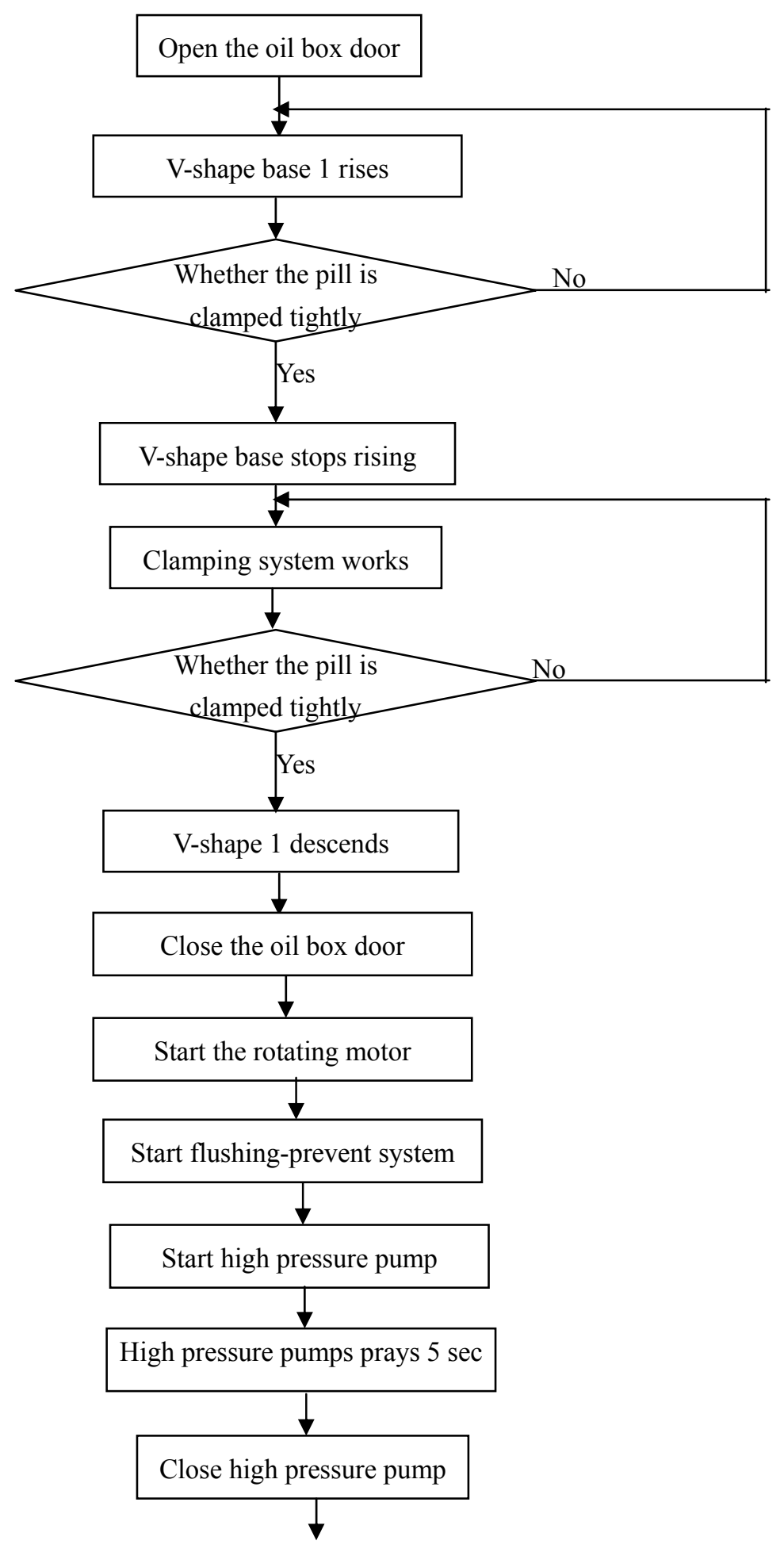




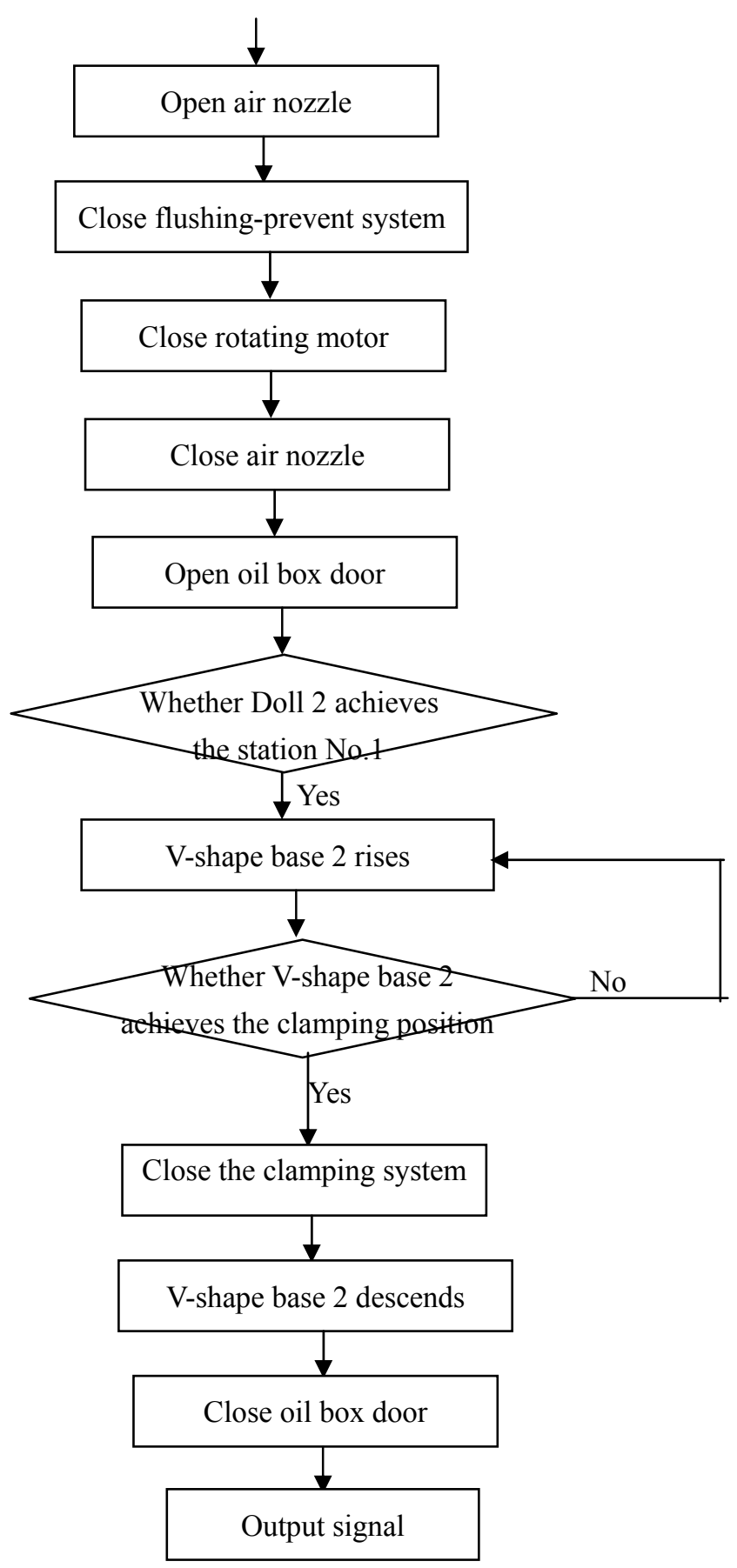

Figure 3. Program Flow Chart of Ammunition Degreasing Station Control System 